\title{
Riuscito trattamento di trombosi acuta della vena cava inferiore e di una vena renale con infusione locale di attivatore tessutale ricombinante del plasminogeno
}

\author{
Da: Am J Kidney Dis 1998; 32: 1075-79 \\ KK Lam, CC Lui
}

$\mathrm{U}$ n uomo di 20 anni si ricovera per dolore al fianco sn. L'anamnesi è positiva per sindrome nefrosica da 10 mesi. La biopsia renale dimostra una glomerulonefrite a lesioni minime. Viene instaurata terapia steroidea, con buona risposta clinica. Quando il prednisone viene ridotto a $10 \mathrm{mg} / \mathrm{die}$, insorge steroido-dipendenza. Due settimane prima del ricovero il paziente interrompe la terapia steroidea ed inizia di propria iniziativa un trattamento a base di erbe. Dopo una settimana, episodio di ematuria durato tre giorni. Il dolore al fianco sn insorge il giorno precedente il ricovero.

Alla palpazione, addome trattabile con dolenzia alla regione lombare sn alla palpazione profonda. Marcato dolore all'angolo costo-vertebrale sn. Marcati edemi agli arti inferiori. Tra gli esami di laboratorio, creatinina $1.6 \mathrm{mg} / \mathrm{dl}$, colesterolo $329 \mathrm{mg} / \mathrm{dl}$, protidemia $3.9 \mathrm{~g} / \mathrm{dl}$, albuminemia $1.7 \mathrm{~g} / \mathrm{dl}$, proteinuria $>5 \mathrm{~g} / \mathrm{l}$.

Un esame Doppler dimostra completa assenza di flusso nella vena renale sn. Una risonanza magnetica conferma il referto. Viene iniziata infusione continua di eparina in modo da mantenere il PTT tra 1.5 e 2 volte il valore di riferimento. Dopo 24 ore il dolore persiste. Uno studio angiografico dimostra arterie renali nella norma e ostruzione completa della vena renale sn con il trombo che si estende nella vena cava inferiore. A questo punto viene infuso a livello della vena renale sn attivatore tessutale ricombinante del plasminogeno, alla dose di $15 \mathrm{mg}$ in bolo seguiti da $35 \mathrm{mg}$ nel corso dei successivi 60 minuti. Subito dopo la somministrazione del bolo al Doppler si rileva il ristabilimento di flusso nella vena renale sn, tuttavia al termine dell'infusione di 60 minuti un venogramma di controllo dimostra re-trombosi. Viene immediatamen-

te ripresa la terapia eparinica (che era stata sospesa 4 ore prima dello studio angiografico) e subito dopo si somministra un secondo ciclo di terapia con attivatore del plasminogeno con le stesse modalità del primo; in seguito la terapia con eparina viene proseguita per 7 giorni, con successivo passaggio a dicumarolici. Immediatamente dopo il secondo ciclo di terapia con attivatore del plasminogeno un venogramma di controllo dimostra flusso nella vena renale sn e il dolore scompare. La funzione renale continua a peggiorare fino a 4 giorni dopo la trombolisi con un picco massimo di creatinina di $6.1 \mathrm{mg} / \mathrm{dl}$, tuttavia in seguito si verifica una ripresa (creatinina $0.9 \mathrm{mg} / \mathrm{dl}$ a 3 settimane dalla trombolisi). Una scintigrafia renale con clearance frazionata conferma normale funziona renale in entrambi i reni.

L'interesse di questo caso clinico risiede nel riuscito schema di trattamento aggressivo della trombosi venosa renale con utilizzo combinato di eparina e attivatore del plasminogeno. La sola terapia con anticoagulanti, da molti anni in uso, pur risultando in molti casi di grande beneficio, in altri non riesce a risolvere la trombosi in modo rapido e tale da preludere ad una brillante ripresa funzionale. L'associazione di trombolisi e terapia con anticoagulante era già stata descritta come capace di aumentare la percentuale di successi terapeutici. Il caso in questione risulta comunque il primo in letteratura in cui alla terapia anticoagulante è stato associato l'attivatore tessutale del plasminogeno. Come è noto tale agente è potenzialmente in grado di ottenere maggiore efficacia e minori effetti collaterali rispetto ad urochinasi e streptochinasi. La severità del quadro clinico della trombosi della vena renale sembra comunque giustificare una terapia aggressiva. 